\title{
WILEY-VCH
}

\section{Tilted chiral liquid crystal gratings for efficient large-angle diffraction}

Inge Nys*, Migle Stebryte, Yera Ye. Ussembayev, Jeroen Beeckman, Kristiaan Neyts

Dr. Inge Nys, Migle Stebryte, Yera Ye. Ussembayev, Prof. Jeroen Beeckman, Prof. Kristiaan Neyts

Technologiepark Zwijnaarde 126, Ghent, 9052, Belgium

E-mail: inge.nys@ugent.be

Keywords: chiral liquid crystals, polarization gratings, periodic photo-alignment, Bragg reflection

Deflecting light with high efficiency over large angles with thin optical elements is challenging but offers tremendous potential for applications, such as wearable displays and optical communication systems. Compared to the complex production of metasurfaces, the self-organization of liquid crystal (LC) superstructures provides an elegant and flexible way to produce high-quality thin optical components. The periodically varying dielectric tensor in short-pitch chiral LC gives rise to a photonic bandgap, which can be exploited to realize efficient diffractive mirrors in the visible wavelength range. However, large-angle diffractive devices require a small in-plane period, leading to complex self-assembly behavior in the bulk. This work demonstrates that by patterning photo-alignment layers at the surfaces with a period comparable to the chiral pitch, the LC self-assembles into a tilted, defect-free helical structure. The director configuration is calculated by finite element simulations and it is experimentally demonstrated that a single photo-aligned substrate is sufficient to template the tilted chiral structure in the bulk. This structure effectively (88\%) diffracts light over large angles $\left(\sim 46^{\circ}\right)$ and enables novel micrometer-thin $(\sim 3 \mu \mathrm{m})$ optical components that can be produced with an elegant manufacturing process. Due to flexibility of photo-alignment, this process could easily be implemented in emerging photonic applications.

Miniaturized optical components such as diffraction gratings and lenses have become essential in many applications, ranging from optical communication systems to electronic 


\section{WILEY-VCH}

displays and virtual reality glasses. To replace conventional bulky components, flat optics, based on diffraction from sub-wavelength structures, have attracted significant attention. ${ }^{[1]}$ For operation in the visible wavelength range, the fabrication of metasurfaces with high resolution nanostructures is challenging and the optical quality is not always satisfying. Over the past decade, the use of thin layers of patterned liquid crystal (LC) has become popular for the production of miniaturized optical components. ${ }^{[2-11]}$ These so-called LC optical axis gratings or polarization gratings (PGs) with non-uniform spatial orientation of the optical axis, modulate the geometric phase or Pancharatnam-Berry $(\mathrm{PB})$ phase ${ }^{[12]}$ This is in contrast to conventional volume holographic gratings or surface relief gratings that modulate the dynamic phase. LC PGs combine polarization sensitivity with high single-order diffraction efficiency and enable large diffraction angles in micrometer-thin films. ${ }^{[2-10]}$ In so-called circular nematic liquid crystal (NLC) PGs, the director is rotating while remaining parallel to the plane of the substrate (Figure 1(a)) and is in good approximation uniform over the thickness. ${ }^{[4]}$ This type of PG with spatially varying orientation of the anisotropy axis is obtained in a NLC cell in which the rotating photo-alignment pattern at the top and bottom substrate is identical. The alignment at the substrates, represented by the director field $\mathbf{n}(\mathrm{x}, \mathrm{y})=\sin (\pi \mathrm{x} / \Lambda) \mathbf{1}_{\mathrm{x}}+\cos (\pi \mathrm{x} / \Lambda) \mathbf{1}_{\mathrm{y}}$, can be obtained by holographic exposure of the alignment layers. ${ }^{[4 b, 4 c]}$

With the help of NLC, very efficient polarization-sensitive diffraction has been demonstrated, but the bandwidth is restricted by chromatic dispersion while the efficiency for large angles remains limited. ${ }^{[4-6]}$ To realize achromatic gratings, a dual-twist design has been proposed, in which the $\mathrm{LC}$ is not only rotating in-plane (due to the surface anchoring) but also out-of-plane. ${ }^{[5]}$ This additional periodicity along the cell-normal is induced by adding a chiral dopant to the LC. Thanks to the chirality, the director self-assembles into a periodic helical structure with a certain handedness and period. The spatial period over which the director rotates over $2 \pi$ is called the pitch $p$. Oh et al. demonstrated that achromatic diffraction can be 


\section{WILEY-VCH}

obtained by stacking two long-pitch chiral PGs with opposite twist sense, so that the chromatic dispersion is compensated by the reversed-twist. ${ }^{[5 a]}$ This work attracted significant attention and the dual-twist design was adopted to realize devices with high diffraction efficiency for a wide range of incident angles and to improve the efficiency for large deflection angles in transmissive devices. ${ }^{[4 \mathrm{~d}, 5]}$

Instead of using long-pitch CLC to adjust the diffraction properties of PGs, also shortpitch CLC $(p<1 \mu \mathrm{m})$ can be used in combination with patterned planar anchoring to obtain excellent diffractive components. ${ }^{[2,3,6-9]}$ The intrinsic periodic variation of the director and the associated optical tensor in short-pitch CLC leads to a photonic bandgap which can be exploited to realize efficient polarization-sensitive diffractive devices, with large operation angles in the visible wavelength range. Responsiveness of these PGs to multiple stimuli has been recently demonstrated ${ }^{[8]}$ Unlike the previously described PGs, these gratings work in the thick Bragg regime, making use of the efficient reflection of light within the photonic bandgap. Kobashi et al. demonstrated that planar optical elements with control over the reflected wavefront can be created by patterning the spatial phase of the helical structure, in this way changing the phase of light that is reflected. ${ }^{[2]}$ They successfully fabricated a reflective deflector and Fresnel lens by combining CLC with patterned planar anchoring at the substrates, but the deflection angle was limited $\left(0.45^{\circ}\right)$ because of limitations in patterning resolution. ${ }^{[2]}$ This was resolved by using holographic exposure in a more recent publication but only a limited diffraction efficiency ( $30 \%$ for circularly polarized light) was obtained. ${ }^{[3]}$ The reflective device was produced by spin-coating one $4-\mu \mathrm{m}$-thick layer on top of a photoaligned substrate. To obtain more efficient short-pitch CLC PGs with large diffraction angles, the use of multilayer spin-coating has been reported. ${ }^{[6]}$ A thin layer with helical twist in the direction perpendicular to the substrate is obtained by spin-coating CLC on top of a photoaligned substrate. After spin-coating, the layer is polymerized and the process is repeated until the thickness is sufficient to obtain strong Bragg diffraction. The resulting director 


\section{WILEY-VCH}

configuration is schematically illustrated in Figure 1(d). Deposition of multiple layers, with subsequent layers self-aligning on top of each other, is necessary to obtain planar orientation in the bulk. ${ }^{[6]}$ Although very efficient deflectors have been demonstrated, the lengthy and complicated production process limits the applicability.

In this work we propose a simple and efficient production process to obtain highlyefficient polarization-sensitive reflective diffraction at large angles with short pitch CLC. The gratings are not produced by spin-coating multiple layers on top of a photo-aligned substrate, but by defining a photo-alignment pattern on one or both substrates of a cell. A grating with sufficient thickness is immediately obtained after filling the cell with short pitch CLC. The fabrication process is similar as described by Kobashi et al. but we use interference of two circularly polarized laser beams in the photo-alignment procedure, leading to a period that is almost 100 times smaller, and diffraction angles that are 100 times larger. ${ }^{[2]}$ These large diffraction angles are obtained with high diffraction efficiency and the director configuration near the interface and in the bulk is explained in detail. Moreover, we demonstrate that periodic photo-alignment at the front substrate is sufficient to template the tilted chiral structure and obtain efficient diffraction in a cell with homeotropic alignment at the back substrate. Defects are present close to the homeotropic substrate but hardly influence the diffraction when the cell is sufficiently thick $(\sim 5 \mu \mathrm{m})$.

In the bulk of a conventional NLC PG, the director follows the alignment at the substrates (approximately independent of the z-coordinate) as long as the cell thickness is small enough with respect to the alignment period. ${ }^{[4]}$ When the same type of rotating planar alignment (Figure 1(a)) is used to align CLC, the director configuration depends not only on the cell thickness $d$ and alignment period $\Lambda$ but also on the chiral pitch $p$ and the handedness of the CLC. When $p$ is much smaller than $\Lambda$, a quasi-standing helix structure is formed with a spatially varying helix phase as has been described by Kobashi et al. ${ }^{[2]}$ We here report that, when $p$ becomes comparable to $\Lambda$, a very different situation is obtained, in which the helix 


\section{WILEY-VCH}

axis is tilted over an angle $\alpha$ compared to the substrate normal (Figure 1(b,c)). The value of $\alpha$ is defined by the parameters $p, \Lambda, d$, while the direction in which the helix axis deviates from the cell-normal is defined by the handedness of the CLC. Note that Xiang et. al. suggested that a non-planar (with out-of-plane tilt) director profile may occur in PGs produced with the repeated spin-coating method. They did not determine the tilt angle and assumed it to be constant everywhere. ${ }^{[\mathrm{b}]}$ This does not correspond with the director profile that we obtain from simulation for our device. Based on experimental observation, Lee et al. suggested the existence of a slanted instead of planar PG grating in spin-coated samples. ${ }^{[\mathrm{b}]}$ They propose that for certain slant angles a slanted structure might have minimal free energy. Our simulations show that in cells with photo-aligned substrates, a slanted structure has a lower energy than a planar structure and that the tilt angle is not constant.

In the first experiment reported here, we combine two photo-aligned substrates with alignment period $\Lambda=0.88 \mu \mathrm{m}$, a cell thickness $d=3 \mu \mathrm{m}$ and a chiral pitch $p \approx 400 \mathrm{~nm}$. In the second experiment, we combine one substrate with a photo-alignment layer and one with a homeotropic alignment layer. The alignment period in this cell is $\Lambda=1.1 \mu \mathrm{m}$, the cell thickness $d=5.5 \mu \mathrm{m}$ and the chiral pitch $p \approx 400 \mathrm{~nm}$. As shown in the polarizing optical microscopy (POM) images for the cell with two photo-alignment layers in Figure 2(a-d), a rather homogeneous structure is obtained in the region with periodic photo-alignment at both substrates. The periodicity $\Lambda$ of the alignment pattern can just be discerned in Figure $2(b, c)$ (it is more clearly visible in reflection, see Figure S1). Just after filling the cell and rapidly cooling down to room temperature, two areas with different color appear (Figure 2(a)). However, after storing the cell in a dark environment for a few hours, only one homogeneous region remains (Figure 2(d)). This is reminiscent of the behaviour of CLC in cells with uniform planar alignment, with domains having different numbers of half pitches. The POM images for the cell with one homeotropic alignment layer show a different behaviour: no domains with different color are present but a distorted, spotted structure is visible in the 


\section{WILEY-VCH}

transmission images (Figure 2(e) and 2(f)). A CLC structure is incompatible with uniform homeotropic anchoring and as a result defects are formed. A strongly distorted director configuration is present close to the homeotropic substrate as is evident from Figure 2(g) and 2(h). When this cell is observed in reflection with the photo-aligned substrate oriented towards the illumination, a uniform clean image appears for the circular polarization that is reflected by the CLC (Figure 2(h)) while a non-uniform image with distortions is observed for the opposite circular polarization (Figure 2(g)). Since the LC layer is sufficiently thick the distortions close to the homeotropic surface hardly influence the reflection inside the photonic bandgap.

To obtain detailed information about the director configuration in the bulk of the device numerical simulations have been performed. In the finite element simulation software we use, the $\mathrm{LC}$ is represented by a rank two order tensor (Q-tensor) and the spatial distribution of the director is found by minimizing the Landau-de Gennes free-energy. ${ }^{[13]} \mathrm{We}$ focus on the simulation of the cell with two patterned photo-alignment layers, with a defectfree director configuration. Periodically rotating alignment conditions at the top and bottom interfaces are included using strong anchoring conditions. Details about the simulation can be found in previous references and in the supporting information. ${ }^{[13]}$ The simulation results are summarized in Figure 3 for left-handed CLC. For right-handed material, the helical axis would be mirrored with respect to the yz-plane. In the bulk the director configuration corresponds to a regular helical CLC structure with the helix axis tilted away from the cell normal by an angle $\alpha$. Minimization of the elastic energy in the bulk is the driving force to organize this structure: the regular helical CLC structure in the bulk of Figure 3, with all directors perpendicular to the helical axis, contains no elastic energy. There is only the twist corresponding to the chirality of the material. This is in contrast to the dual-twist configuration (Figure 1(d)) of spin-coated CLC layers, in which the helical axis is not perpendicular to the slanted planes of the grating and the structure contains splay and bend. In 


\section{WILEY-VCH}

our configuration, close to the top- and bottom substrates, the structure is adjusted to match the anchoring conditions. No energetically costly defects are required and a smooth transition from the rotating surface alignment to the bulk CLC configuration is obtained (Figure 3(d)). This illustrates that the periodic surface anchoring is very well-suited to stabilize a slanted CLC structure.

We can obtain an (approximate) relation between the inclination angle $\alpha$ and the cell parameters $\Lambda$ and $d$ by assuming that the slanted structure extends over the entire thickness:

$s \cdot \Lambda \cdot \tan (\alpha)=d$

with $s$ an integer representing the number of slanted layers that are present in a vertical section (Figure $3(\mathrm{a})$ ). For a fixed alignment period $\Lambda$, only a discrete number of inclination angles $\alpha$ is allowed since an integer number of layers $s$ has to fit in the cell thickness. The value for $s$, and therefore also the inclination angle $\alpha$, is fixed by the requirement to have minimal elastic energy in the structure. More specifically, to minimize the elastic energy in the bulk the value of $s$ will be such that the resulting pitch in the structure $p$ ' is close to the pitch $p$ of the material (Figure 3(a)):

$p^{\prime}=2 \cdot \Lambda \cdot \sin (\alpha)=\frac{2}{\sqrt{\frac{s^{2}}{d^{2}}+\frac{1}{\Lambda^{2}}}} \approx p$

Sometimes consecutive values of $s$, can almost equally well satisfy Equation 2 which gives rise to two possible states with slightly different inclination angles and very similar values for the total elastic energy. The transition region between different states involves a defect line, which tends to move towards the domain with higher energy. This is what is observed experimentally after filling the cell with two photo-alignment layers: domains with different optical transmission represent regions with different values of $s$, different orientations of the helix axis and different periods $p$ '. The domain with highest free energy is metastable and slowly disappears (Figure 2). 


\section{WILEY-VCH}

For the cell with one homeotropic alignment layer, the condition expressed by Equation 1 disappears and in Equation 2 ' becomes equal to $p$. The photo-aligned substrate templates the tilted chiral structure in the bulk, while close to the homeotropically aligned substrate distortions occur to accommodate the anchoring.

From Equation 2 it follows that when the pitch $p$ is much smaller than the period $\Lambda$, a quasi-standing helix structure $\left(\alpha \approx 0^{\circ}\right)$ is formed, with varying helix phase (Figure $1(\mathrm{~d})$ ). When the period $\Lambda$ becomes comparable to the pitch $p$, as is the case here, the helix axis substantially tilts away from the cell normal (Figure 3(a)). In the bulk of the cell an undisturbed CLC helix with zero elastic energy is formed, in which the LC director remains perpendicular to the tilted helix axis. The director tilt angle, measured between the director and the $x y$-plane, is fluctuating as shown in Figure 3(b). The director structure resembles a slanted grating with alternating refractive index planes and shows similarities to the dual-twist PGs proposed previously (Figure 1(d)). ${ }^{[6]}$ However, in the dual-twist structure, obtained by deposition and polymerization of multiple thin CLC layers, the director ideally remains planar (tilt angle close to $0^{\circ}$ ), which means that the directors are not perpendicular to the slanted planes of the grating. This standing helix structure with variable helix phase gives rise to splay and bend in the LC layer with corresponding elastic energies densities respectively $\frac{1}{2} K_{11}(\nabla \cdot \mathbf{n})^{2}=\frac{1}{2} K_{11} \frac{\pi^{2}}{\Lambda^{2}} \cos ^{2}\left(\frac{\pi}{\Lambda}\left(x+\frac{2 \Lambda z}{p}\right)\right)$ and $\frac{1}{2} K_{33}(\mathbf{n} \times(\nabla \times \mathbf{n}))^{2}=\frac{1}{2} K_{33} \frac{\pi^{2}}{\Lambda^{2}} \sin ^{2}\left(\frac{\pi}{\Lambda}(x+\right.$ $\left.\left.\frac{2 \Lambda z}{p}\right)\right)$. This configuration is therefore unfavorable if thick layers are spin-coated or when a photo-patterned cell is filled with CLC.

The tilted chiral structure formed in our cells yields very efficient diffraction. It operates in the thick Bragg grating regime and acts as a polarization-sensitive reflective grating with large diffraction angle. The diffraction angles $\theta_{\text {out }}$ with respect to the substrate normal is determined by the grating equation, stating that the difference in the $k$-vector for 
incoming and outgoing light projected on the $x y$-plane, should be a multiple of the $k$-vector of the grating:

$n_{\text {out }} \sin \left(\theta_{\text {out }}\right)=\frac{m \lambda}{\Lambda}+n_{\text {in }} \sin \left(\theta_{\text {in }}\right)$

with $m$ an integer number. For light that is incident perpendicular to the substrate $\left(\theta_{\text {in }}=0^{\circ}\right)$ the last term vanishes. Remark that according to Equation (3) the possible diffraction angles depend only on the period $\Lambda$ of the anchoring pattern, and not on the pitch $p$ or the slant angle $\alpha$.

In the cell with two photo-alignment layers with alignment period $\Lambda=0.88 \mu \mathrm{m}$ only the first diffraction order $(m=1)$ is observed for perpendicular incidence and the diffraction angle in air $\theta_{\text {out }}$ for red light $(\lambda=633 \mathrm{~nm})$ is $46^{\circ}$. In the cell with one homeotropic alignment layer and with alignment period $\Lambda=1.1 \mu \mathrm{m}$ at the photo-aligned substrate the corresponding diffraction angle for perpendicular incidence in air is $35^{\circ}$ for red light. An image illustrating the diffraction of unpolarized white light is shown in Figure 3(e): the first diffraction order is clearly visible on the right (with separate red, green and blue spots), while the order $m=-1$, that should appear on the left is absent. The $1^{\text {st }}$ order diffraction efficiency and the transmission at perpendicular incidence were measured for a He-Ne laser $(\lambda=633 \mathrm{~nm})$ for left- and right-handed circular polarization. For the cell with two photo-alignment layers, upon inversion of the handedness of the circular polarization, the power in the $1^{\text {st }}$ diffracted order decreased from $86 \%$ to $3 \%$ while the transmission through the cell increased from $2 \%$ to $90 \%$ (w.r.t. the incident power). For the cell with one homeotropic alignment layer, upon inversion of the handedness of the circular polarization, the power in the $1^{\text {st }}$ diffracted order decreased from $77 \%$ to $2 \%$ while the transmission through the cell increased from $1 \%$ to $85 \%$ (w.r.t. the incident power).This illustrates that the gratings are strongly selective to the input polarization and that incident light with the same handedness as the CLC is efficiently diffracted. A high $1^{\text {st }}$ order diffraction efficiency with a large diffraction angle can be obtained 


\section{WILEY-VCH}

in thin cells $(d=3 \mu \mathrm{m}$ and $d=5.5 \mu \mathrm{m}$ in the cell with two and one photo-alignment layers respectively). The device performance (efficiency, angle and polarization sensitivity) is superior to what can be obtained with conventional NLC PGs and is comparable to what was reported previously for PGs with a dual-twist design. Our devices are however produced with a very simple one-step process, avoiding the need to spin-coat and polymerize multiple thin layers. A previously reported attempt to make a CLC PG with large reflection angle by spincoating only one layer, resulted in a much lower diffraction efficiency of $\sim 30 \%$ for circularly polarized light. ${ }^{[3]}$

Remark that although the chiral pitch does not influence the diffraction angle $\theta_{\text {out, }}$, it is important for the diffraction efficiency. The chiral pitch defines the grating slant and therefore the diffraction efficiency as a function of the incident angle. Only light inside the photonic bandgap and with circular polarization of the same handedness as the CLC is effectively reflected. For a plane wave propagating at an angle $\beta$ with respect to the helix axis, the bandedges are shifted to the blue side of the spectrum. A high diffraction efficiency is expected when the wavelength of the incident light is inside the interval $n_{o} p \cos (\beta)<\lambda<n_{\mathrm{e}} p$ $\cos (\beta)$. Additionally, the device should contain enough chiral periods to obtain efficient Bragg diffraction. A larger thickness is required in a cell with one photo-aligned and one homeotropic substrate to avoid influence of the distortions close to the homeotropic substrate. To estimate the diffraction efficiency a numerical simulation of the light propagation is necessary. We performed a finite element method (FEM) calculation of the diffraction properties with the commercially available COMSOL simulation tool. The results, obtained for the cell with two photo-alignment layers and the director configuration of Figure 3a, are summarized in Figure 4. More details about the FEM calculation can be found in the supporting information. Upon inversion of the handedness of the circular polarization, the calculated power in the $1^{\text {st }}$ diffracted order at $\lambda=633 \mathrm{~nm}$ decreased from $98.0 \%$ to $0.1 \%$ while the transmission through the cell increased from $0.7 \%$ to $98.7 \%$. This is in reasonable 


\section{WILEY-VCH}

agreement with the experimentally obtained values, if one realized that reflection losses at the air-glass interfaces ( 4\%) and scattering are not taken into account in the simulations. Figure 4(c) clearly illustrates how the existence of a photonic bandgap in the visible wavelength range influences the diffraction behavior as a function of the wavelength. The spectral range of high reflectivity is limited by the width of the photonic bandgap and therefore by the birefringence of the LC. To extend the spectral range, a second cell with a CLC material with a different pitch could be added.

In conclusion, we can state that we have developed CLC diffraction gratings based on a simple manufacturing process, with high polarization-sensitivity, large diffraction angle and high $1^{\text {st }}$ order diffraction efficiency. Experimentally, we measured a large diffraction angle $\left(\sim 46^{\circ}\right.$ and $\sim 35^{\circ}$ at $\left.\lambda=633 \mathrm{~nm}\right)$ and a high diffraction efficiency $(\sim 86 \%$ and $\sim 77 \%)$ with strong polarization sensitivity. The devices provide an excellent balance between efficiency, spectral bandwidth and viability of the manufacturing process. The director configuration has been numerically simulated with the help of a finite element model and COMSOL Multiphysics has been used to simulate the diffraction properties. In cells with a short alignment period, and accordingly a large diffraction angle, the self-assembled helical structure has a strongly tilted helical axis. The periodic structure at the interface can be matched perfectly with the chiral pitch by providing an appropriate slant angle. Elastic energy is only present in a small transition region between the interface and the bulk, and as a result thick CLC layers with high quality periodicity have minimal energy and are easily obtained. The fact that a one-step filling process is sufficient to obtain a device with excellent characteristics implies that this approach is ideally suited for non-mechanical beam-steering and for waveguide-based headmounted displays. ${ }^{[6 \mathrm{~b}, 6 \mathrm{~d}, 7-10]}$ 


\section{WILEY-VCH}

\section{Experimental Section}

Sample Preparation: The cell with two photo-alignment layers consists of two indium tin oxide (ITO) coated glass substrates (Delta Technologies, ITO coated float glass $1.1 \mathrm{~mm}$ ), each covered with a photo-alignment layer PAAD 22 (photo-aligning azo dye, Beamco). The photo-alignment layer is spin-coated on the glass substrates (3000 rpm, $30 \mathrm{~s})$ and the solvent is evaporated on a hotplate $\left(90^{\circ} \mathrm{C}, 10 \mathrm{~min}\right)$. The second cell consists of one substrate covered with photo-alignment layer (Brilliant Yellow, Sigma Aldrich) and one with homeotropic alignment material (SE4811, Nissan Chemical Industries). The homeotropic layer is spincoated on the glass substrate $(2500 \mathrm{rpm}, 15 \mathrm{~s})$, tempered for $2 \mathrm{~min}$ at $90^{\circ} \mathrm{C}$ on a hot plate and baked for $1 \mathrm{~h}$ at $180^{\circ} \mathrm{C}$ in the oven. The substrates are glued together using a glue (NOA68) with spherical spacer balls near the edges. After gluing the cell is illuminated with a UV interference pattern of right- and left-handed circularly polarized light. For the illumination a continuous wave UV laser emitting at $355 \mathrm{~nm}$ (Coherent, Genesis CX SLM, 100mW) is used. In the experiment a mixture of the NLC E7 ( 95.5 wt\%) and the chiral dopant BDH 1305 $(\sim 4.5 \mathrm{wt} \%)$ is used, resulting in a chiral pitch $p \approx 0.4 \mu \mathrm{m}$. The cells are filled above the isotropic-nematic transition temperature and cooled down to room-temperature. The microscope images are made with a polarizing optical microscope (Nikon Eclipse E400 POL). To analyze the diffraction, a continuous wave He-Ne laser (JSD uniphase, $633 \mathrm{~nm}, 4 \mathrm{~mW}$ ) is used and the diffraction efficiency is detected with a power meter (Newport 2936C). The HeNe light source is linearly polarized and the polarization state is controlled by manually rotating a quarter waveplate.

\section{Supporting Information}

Supporting Information is available from the Wiley Online Library or from the author.

\section{Acknowledgements}

((Acknowledgements, general annotations, funding. Other references to the title/authors can also appear here, such as "Author 1 and Author 2 contributed equally to this work.")) 


\section{WILEY-VCH}

Received: ((will be filled in by the editorial staff))

Revised: ((will be filled in by the editorial staff)) Published online: ((will be filled in by the editorial staff))

\section{References}

[1] a) N. Yu, F. Capasso, Nat. Mater. 2014, 13, 139; b) G. Zheng, H. Mühlenbernd, M.

Kenney, G. Li, T. Zentgraf, S. Zhang, Nat. Nanotechnol.2015, 10, 308; c)

R. C. Devlin, M. Khorasaninejad, W. T. Chen, J. Oh, F. Capasso, Proc. Natl. Acad. Sci. USA. 2016, 113, 10473.

[2] J. Kobashi, H. Yoshida, M. Ozaki, Nat. Photon. 2016, 10, 389.

[3] J. Kobashi, Y. Mohri, H. Yoshida, M. Ozaki, Opt. Data Process. Storage 2017, 3, 61.

[4] a) W. Duan, P. Chen, B.-Y. Wei, S.-J. Ge, X. Liang, W. Hu, Y.-Q. Lu, Opt. Mater.

Express 2016, 6, 597; b) C. Provenzano, P. Pagliusi, G. Cipparrone, Appl. Phys. Lett. 2006, 89, 121105; c) G. P. Crawford, J. N. Eakin, M. D. Radcliffe, A. Callan-Jones, R. A. Pelcovits, J. Appl. Phys. 2005, 98, 123102; d) T. Li, Doctor of Philosophy, The Hong Kong University of Science and Technology, August, 2013.

[5] a) C. Oh, M. J. Escuti, Opt. Lett. 2008, 33, 2287; b) H. Cheng, A. K. Bhowmik, P. J. Bos, Appl. Opt. 2015, 54, 10035; c) H. Cheng, A. K. Bhowmik, P. J. Bos, Opt. Lett. 2015, 40, 2080; d) X. Xiang, J. Kim, R. Komanduri, M. J. Escuti, Opt. Express 2017, 25, 19298; e) Gao,

C. McGinty, H. Payson, S. Berry, J. Vornehm, V. Finnemeyer, B. Roberts, P. Bos, Opt. Express 2017, 25, 6283.

[6] a) X. Xiang, J. Kim, M. J. Escuti, Sci. Rep. 2018, 8, 10; b) Y. Lee, K. Yin, S.-T. Wu, Opt. Express 2017, 25, 27008; c) X. Xiang, M. J. Escuti, Proc. Of SPIE 2017, 10127, 10127OD; d) Y. Weng, D. Xu, Y. Zhang, X. Li, S.-T. Wu, Opt. Express 2016, 24, 4588. [7] a) T. Zhan, Y.-H. Lee, G. Tan, J. Xiong, K. Yin, F. Gou, J. Zou, N. Zhang, D. Zhao, J. Yang, S. Liu, S.-T. Wu, J. Opt. Soc. Am. B 2019, 36, D52; b) T. Zhan, J. Xiong, G. Tan, Y.-H. Lee, J. Yang, S. Liu, S.-T. Wu, Opt. Express 2019, 27, 15327. 


\section{WILEY-VCH}

[8] R. Chen, Y.-H. Lee, T. Zhan, K. Yin, Z. An, S.-T. Wu, Adv. Opt. Mater. 2019, 7, 1900101.

[9]

a) Y.-H. Lee, Z. He, S.-T. Wu, J. Opt. Soc. Am. B 2019, 36, D9; b) X. Xiang, M. J.

Escuti, J. Opt. Soc. Am. B 2019, 36, D1.

[10] a) M. Jang, Y. Guo, H. Yu, Z. Zhou, T. Turiv, O. D. Lavrentovich, Q.-H. Wei, Adv.

Mater. 2019, 31, 1808028; b) H. Yu, Z. Zhou, Y. Qi, X. Zhang, Q.-H. Wei, J. Opt. Soc. Am. B 2019, 36, D107.

[11] a) U. Ruiz, P. Pagliusi, C. Provenzano, E. Lepera, G. Cipparone, Appl. Opt. 2015, 54,

3303T; b) Sasaki, R. Shimura, K. Kawai, K. Noda, M. Sakamoto, N. Kawatsuki, H. Ono, Jpn. J. Appl. Phys. 2016, 55, 012001.

[12] a) S. Pancharatnam, Proc.-Indian Acad. Sci. Sect. A 1956, 44, 247; b) M. V. Berry,

Proc. R. Soc. London Ser. A. 1984, 392, 45.

[13] a) R. James, E. Willman, F. A. Fernández, S. E. Day, IEEE Trans. Electron Devices 2006, 53, 1575; b) E. Willman, F. A. Fernández, R. James, S. E. Day, J. Disp. Technol. 2008, 4, 276; c) E. Willman, F. A. Fernandez, R. James, S. E. Day, IEEE Trans. Electron Devices 2007, 54, 2630; d) I. Nys, J. Beeckman, K. Neyts, Soft Matter 2015, 11, 7802; e) I. Nys, K. Chen, J. Beeckman, K. Neyts, Adv. Opt. Mater. 2018, 6, 1701163; f) I. Nys, J. Beeckman, K. Neyts, Adv. Opt. Mater. 2018, 6, 1800070; g) I. Nys, V. Nersesyan, J. Beeckman, K. Neyts, Soft Matter 2018, 14, 6892.

(a) photo-alignment pattern

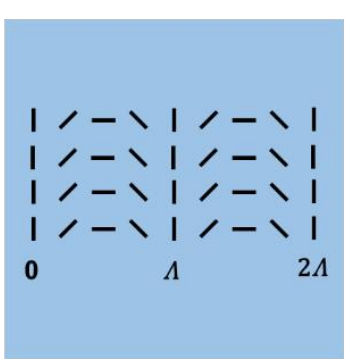

(b) One-step CLC PG

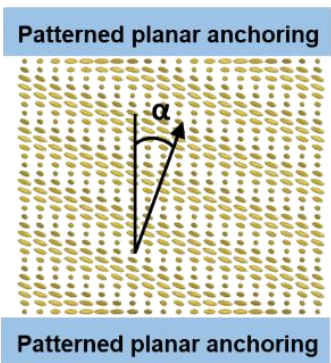

(c) One-step CLC PG

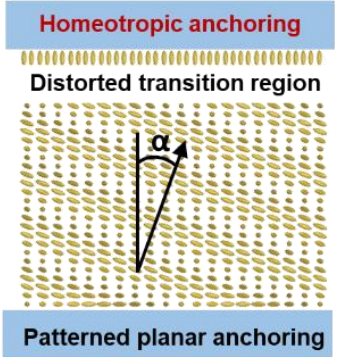

(d) Multi-layer dual-twist PG

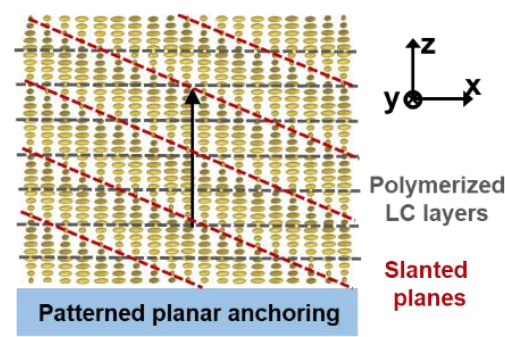

Figure 1. (a) Periodic photo-alignment pattern at the substrates; (b,c) schematic director configuration in a CLC cell with the same photo-alignment pattern at top and bottom substrates (b), with one photo-aligned substrate and one homeotropically-aligned substrate 


\section{WILEY-VCH}

(c); (d) director configuration in a multi-layer dual-twist grating obtained by spin-coating multiple CLC layers on top of a photo-aligned substrate. The helix axis orientation is indicated with a black arrow.
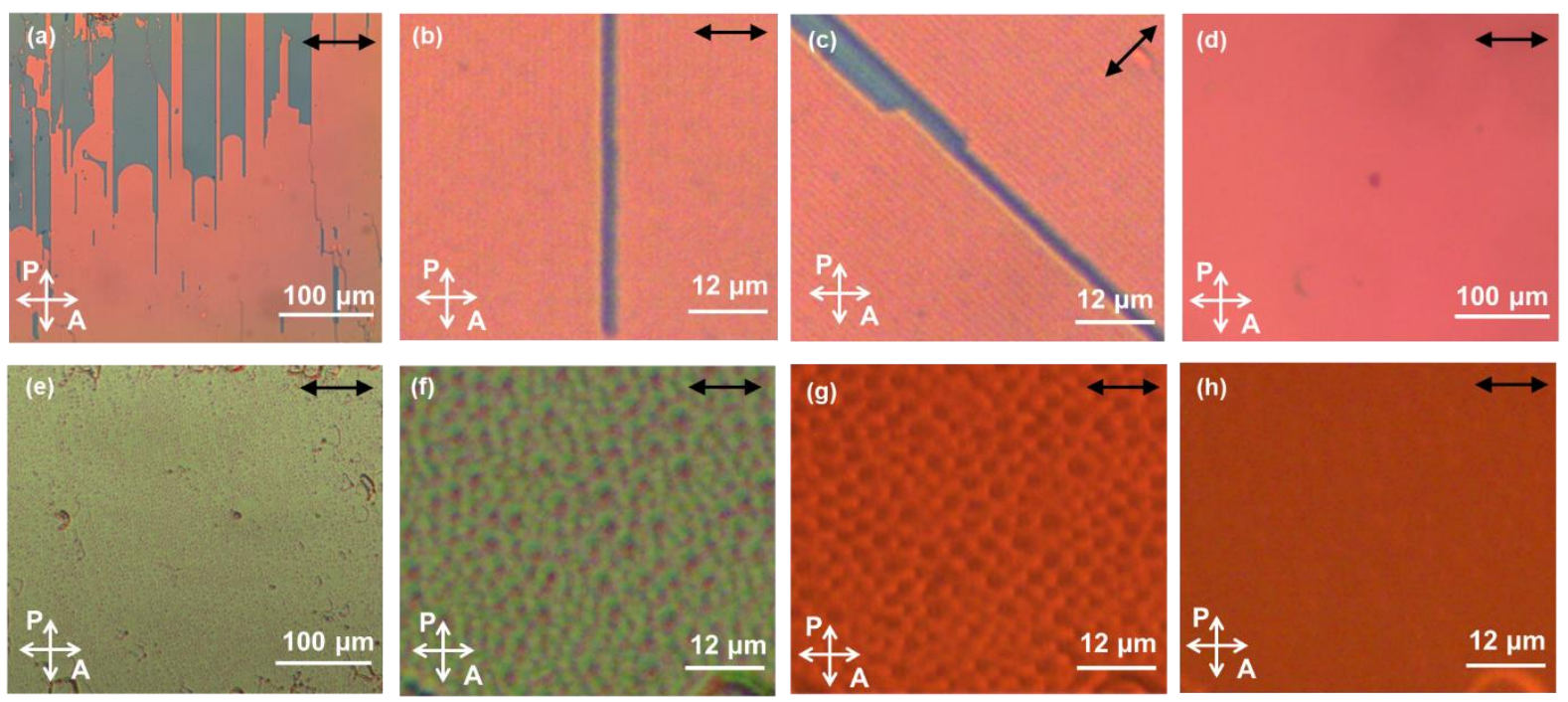

Figure 2. (a-d) POM images in transmission with different magnification inside the photoaligned region of the cell with two photo-alignment layers, thickness $d=3 \mu \mathrm{m}$ and period $\Lambda=$ $0.88 \mu \mathrm{m}$. (e-h) POM images in transmission $(\mathrm{e}, \mathrm{f})$ and reflection $(\mathrm{g}, \mathrm{h})$ inside the photoaligned region of the cell with one homeotropic alignment layer, thickness $d=5.5 \mu \mathrm{m}$ and period $\Lambda=1.1 \mu \mathrm{m}$. The images in reflection $(\mathrm{g}, \mathrm{h})$ are taken with a red filter and the incident polarization is right- and left-circularly polarized in $\mathrm{g}$ and $\mathrm{h}$ respectively. The orientation of the grating vector is indicated with a black arrow. 


\section{WILEY-VCH}

(a)

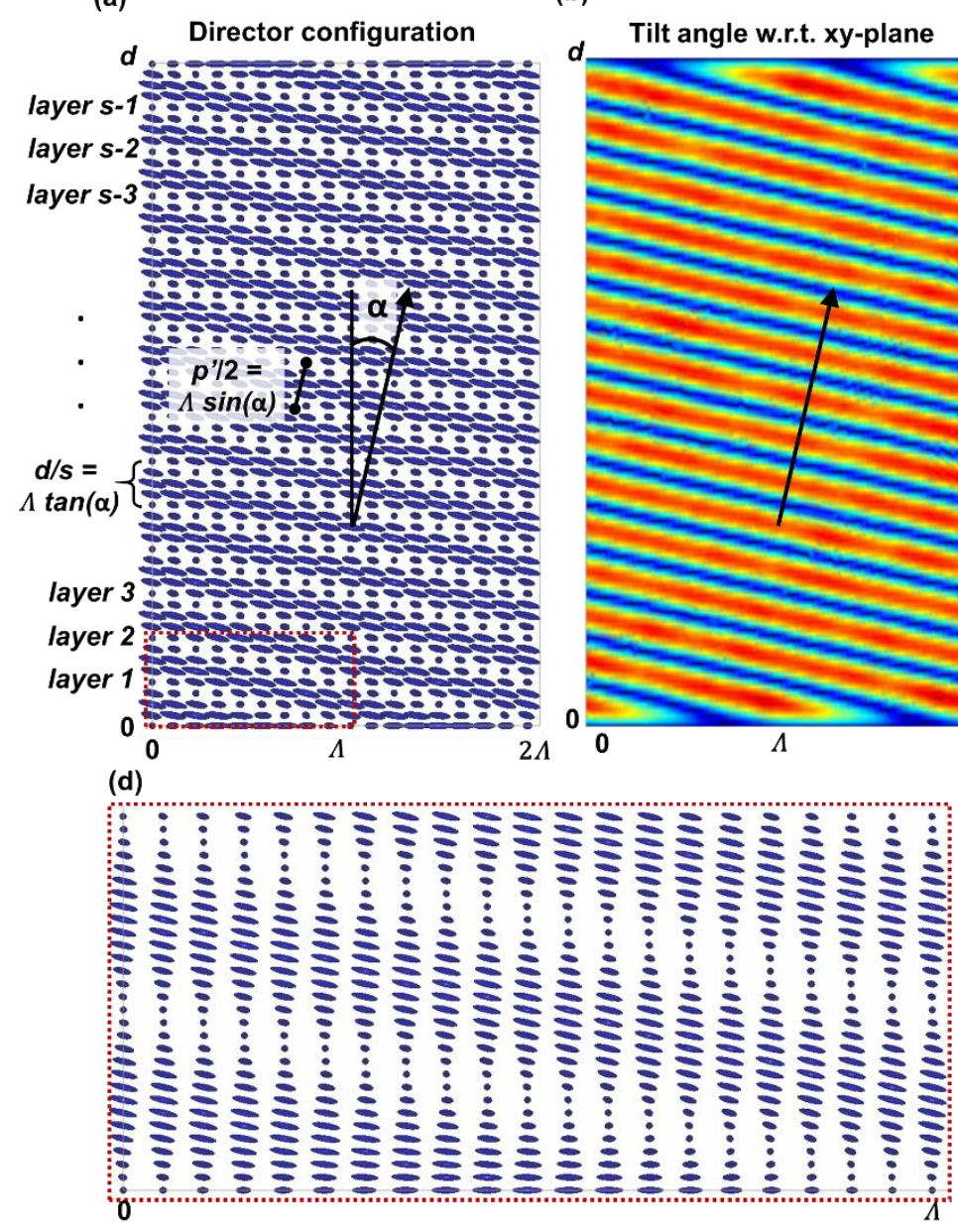

(c)

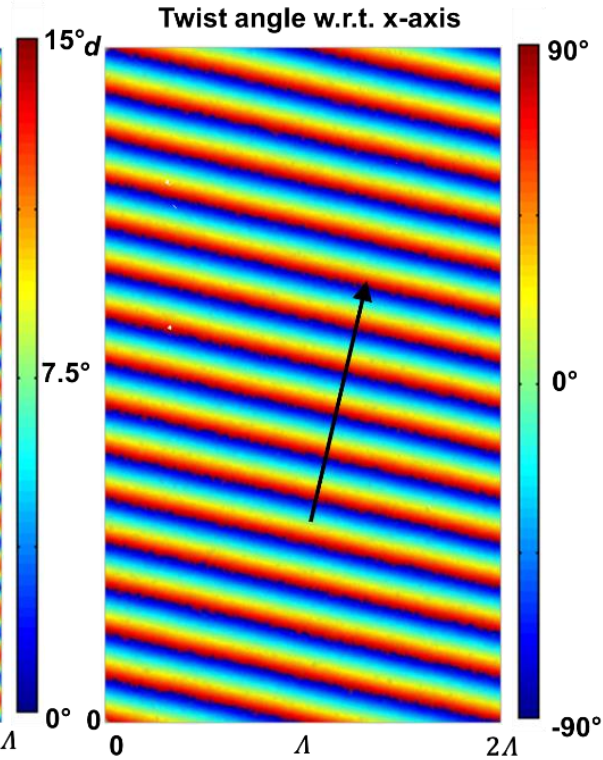

(e)

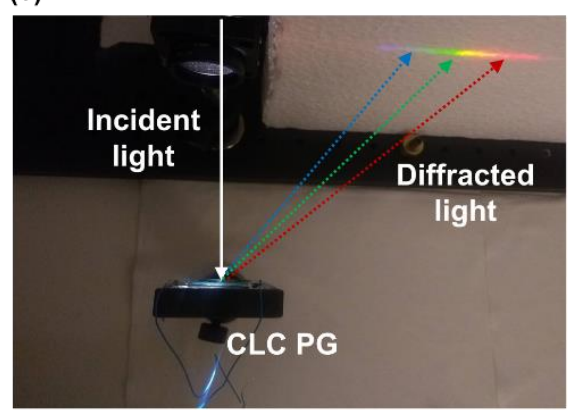

Figure 3. (a-d) Simulated director configuration in a cell with two photo-alignment layers with $\Lambda=0.88 \mu \mathrm{m}, d=3 \mu \mathrm{m}$ and $p=0.4 \mu \mathrm{m}$ for left-handed CLC. The director configuration $(a, d)$ is shown together with a contour plot for the tilt angle (b) and twist angle (c). A detailed view of the director configuration close to the substrate is given in (d). The helical axis orientation is indicated with a black arrow. In this example $s=15$ and $p^{\prime}=0.39 \mu \mathrm{m}$. (e) Optical diffraction for unpolarized white light incident on the cell with periodic photo-alignment at both substrates. 


\section{WILEY-VCH}

(b)
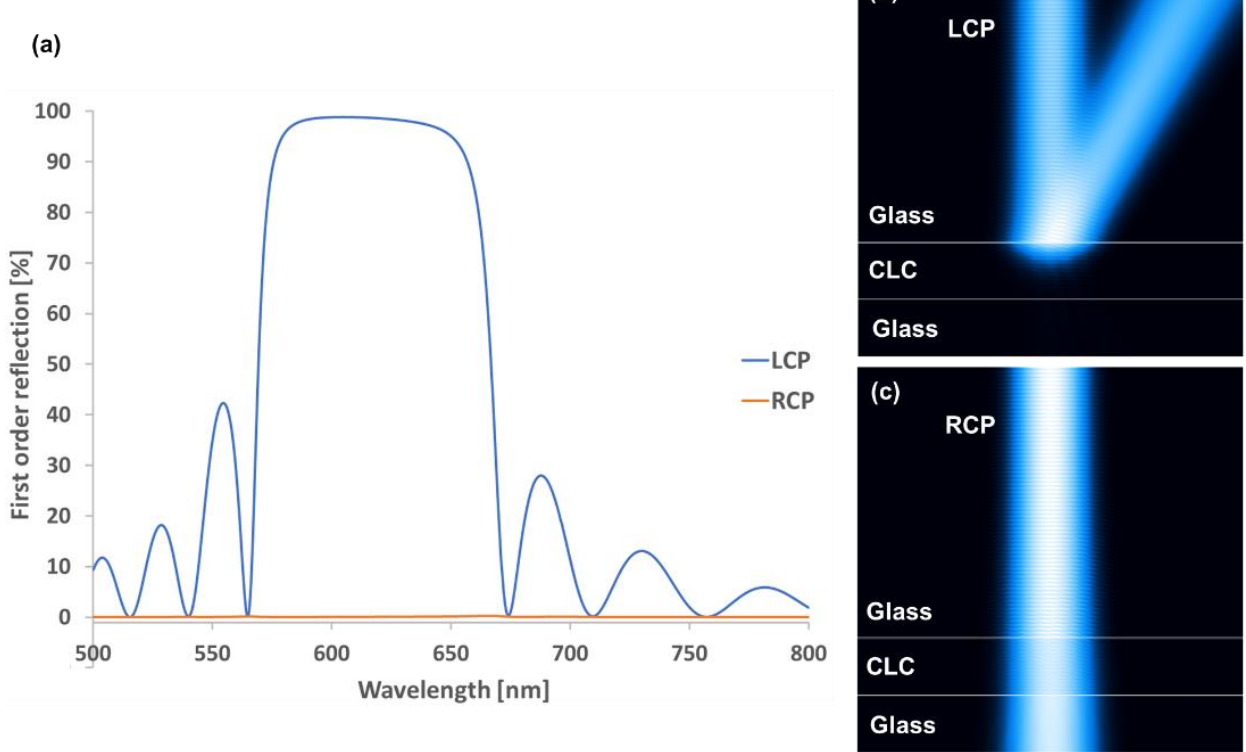

Figure 4. (a) Numerically calculated first order diffraction efficiency spectra for left- and right-handed circularly polarized light (LCP and RCP) under normal incidence in a cell with two photo-aligned layers with $\Lambda=0.88 \mu \mathrm{m}, d=3 \mu \mathrm{m}$ and $p=0.4 \mu \mathrm{m}$. (b,c) Simulated intensity distribution for a left-handed (b) and right-handed (c) circularly polarized Gaussian beam with wavelength $\lambda=633 \mathrm{~nm}$. 


\section{WILEY-VCH}

The table of contents entry should be 50-60 words long and should be written in the present tense and impersonal style (i.e., avoid we). The text should be different from the abstract text.

Self-organization of chiral liquid crystal in cells with photo-patterned surface anchoring leads to the realization of highly-efficient optical components. Micrometer-thin layers efficiently demonstrate large-angle diffraction with strong polarization-sensitivity. Photo-patterning of one or both confining substrates allows to template the tilted chiral bulk structure. The straightforward fabrication process in combination with the versatility of photo-alignment offers great perspectives for emerging photonic applications.

Liquid crystal diffractive optical elements

Inge Nys*, Migle Stebryte, Yera Ye. Ussembayev, Jeroen Beeckman, Kristiaan Neyts

Tilted chiral liquid crystal gratings for efficient large-angle diffraction

ToC figure

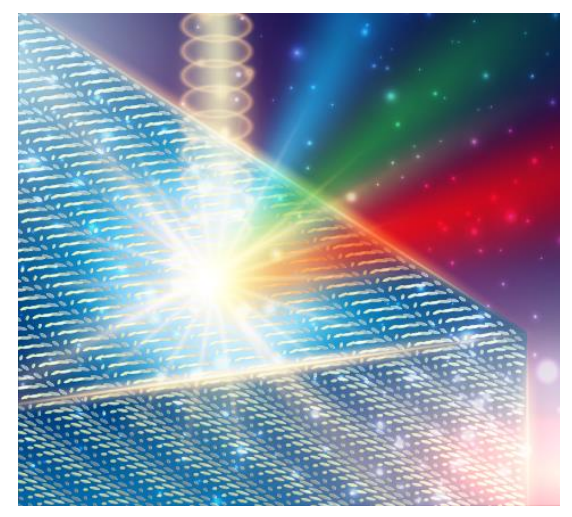

Copyright WILEY-VCH Verlag GmbH \& Co. KGaA, 69469 Weinheim, Germany, 2019. 


\section{WILEY-VCH}

\section{Supporting Information}

\section{Tilted chiral liquid crystal gratings for efficient large-angle diffraction}

Inge Nys*, Migle Stebryte, Yera Ye. Ussembayev, Jeroen Beeckman, Kristiaan Neyts
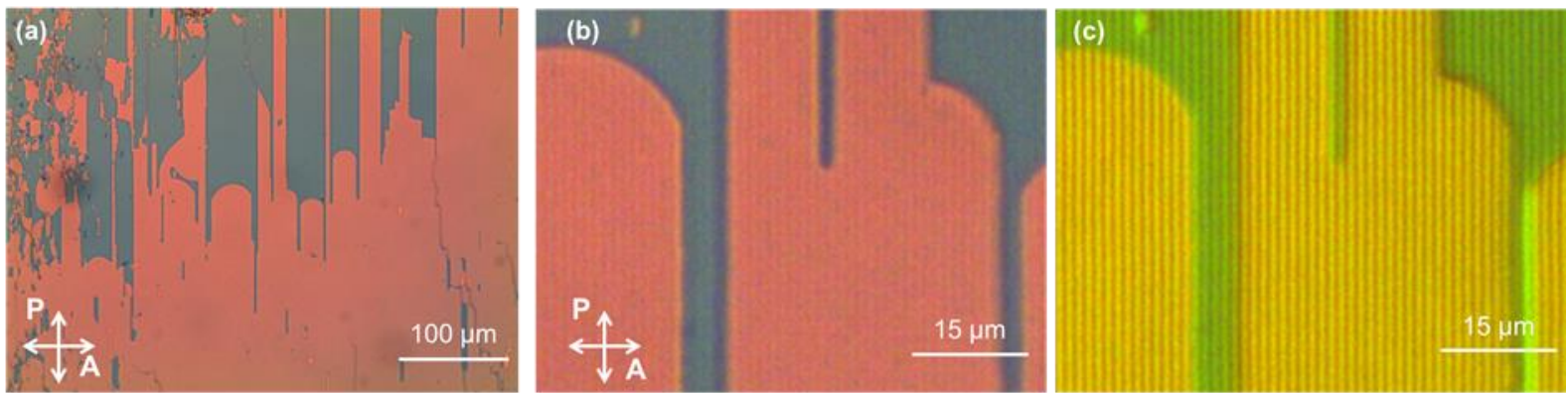

Figure S1. Optical microscopy images in transmission $(a, b)$ and reflection (c) for a cell with $1 \mathrm{D}$ periodic azimuthal photo-alignment at both substrates, filled with short-pitch CLC ( $p \approx 0.4$ $\mu \mathrm{m})$ obtained immediately after filling the cell.
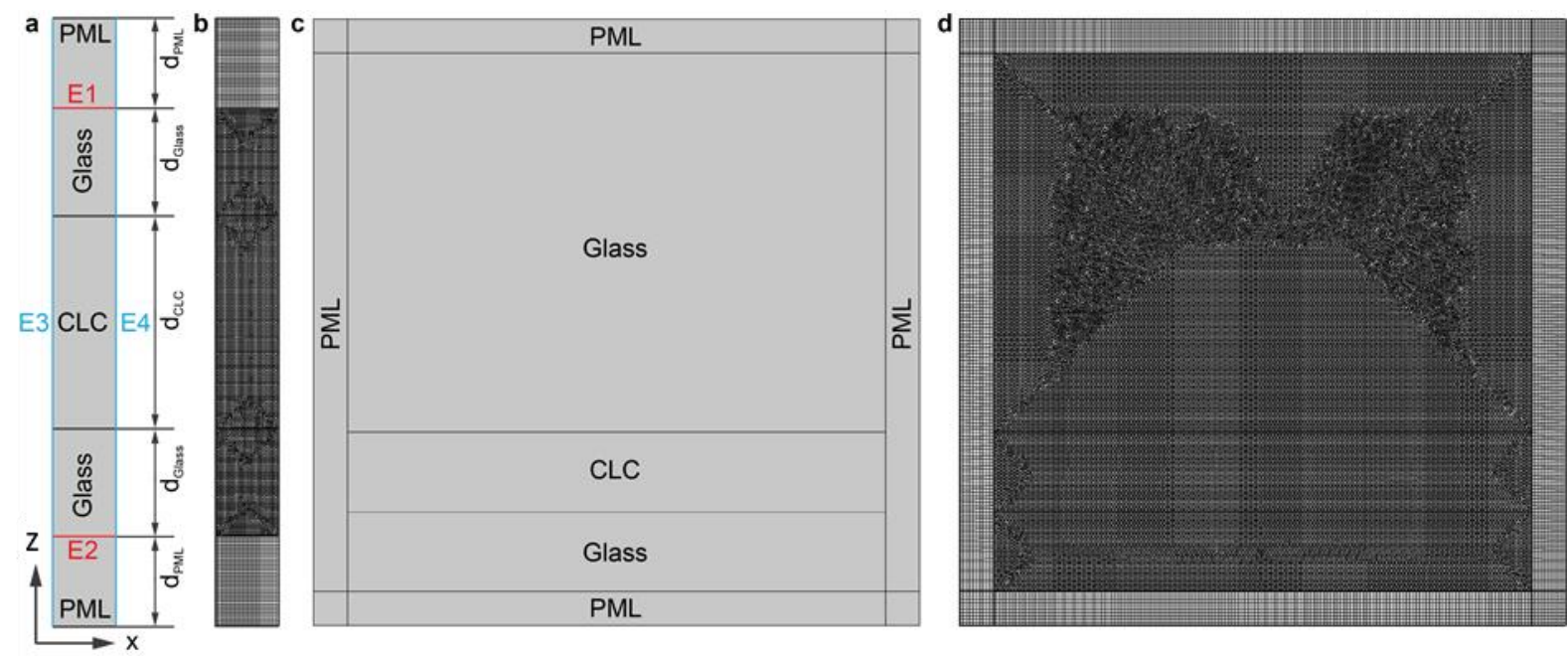

Figure S2. (a) Geometry, materials and boundary conditions (E1 and E2 are port boundary conditions; E3 and E4 are periodic boundary conditions) of the unit cell with a width of $\Lambda$ confined between two perfectly matched layers (PML) for the diffraction efficiency calculations in Figure 4(a). (b) Meshing of this unit cell. (c) Model geometry for a Gaussian beam that is incident on the CLC device as shown in Figures 4(b) and (c). (d) Mesh for this geometry.

\section{Details about the finite element simulations of light interaction with the CLC device:}

FEM-based (Finite Element Modelling) software COMSOL Multiphysics 5.2 (Wave Optics module) is employed to test the light propagation though the CLC cell with numerically calculated director configuration (Figure 4, Main text). Figure S2(a) shows the designed model to study the diffractive properties of the developed CLC structure. The 2D unit cell has a width of one period $\Lambda=880 \mathrm{~nm}$, while the CLC layer with a thickness of $d_{\mathrm{CLC}}=3 \mu \mathrm{m}$ is confined between two glass slabs with $d_{\text {Glass }}=1.5 \mu \mathrm{m}$. The dielectric permittivity tensor components of the liquid crystal are exported from the numerically obtained director structure (Figure 3, Main text), while the refractive index of the glass is set to $n_{\text {Glass }}=1.5$. The port boundary condition at the edge $\mathrm{E} 1$ generates the incident electromagnetic field as $E_{\mathrm{inc}}=\mathrm{E}_{0} \mathrm{e}^{\mathrm{ikz}}$ 


\section{WILEY-VCH}

with an electric field amplitude $\mathrm{E}_{0}$, wave propagation direction along the $z$-axis, a wavevector $k=2 \pi n_{\text {Glass }} / \lambda_{0}$ and a free-space wavelength $\lambda_{0}$. To simulate circularly polarized light, the incident wave is adjusted and has a form $(1, i)$ for left-handed and $(1,-i)$ for right-handed circular polarization. The boundaries E1 and E2 integrate the reflected and transmitted power, respectively, for the $0^{\text {th }}$ and $1^{\text {st }}$ diffraction orders. Periodic boundary conditions are applied for the edges E3 and E4 to simulate an infinitely extended structure. The simulation domain is also confined between two perfectly matched layers (PML) with a typical thickness $d_{\mathrm{PML}}=2$ $\lambda_{0}$ to absorb all the outgoing radiation. The meshing of the model (Figure S2(b)) is performed using a built-in algorithm with a maximum element size of $\lambda_{0} / 12$ and a maximum element growth factor of 1.35 .

Figure S2(c) shows the model geometry applied for the field mapping simulations represented in Figure 4(b) and 4(c) in the main text. The width and height of the calculation domain are 32 $\lambda_{0}\left(\lambda_{0}=633 \mathrm{~nm}\right)$ with the same CLC parameters and dimensions as described in the previous model. The incoming light with circular polarization is described as a weakly-focused Gaussian beam in paraxial approximation with a beam width $w_{0}=3 \lambda_{0}$. The entire domain is enclosed in PML with a thickness of $2 \lambda_{0}$ and discretized into a tetrahedral mesh as exemplified in Figure S2(d).

\section{Details about the finite element Q-tensor simulation tool for the director configuration:}

Our finite element simulation tool uses a continuum theory description in which the $Q$-tensor represents the local director and the order parameter in the LC. The Q-tensor is a symmetric, traceless tensor of rank two and nematic distortions correspond to spatial variations in the eigenvectors and eigenvalues of the Q-tensor. To find the director configuration, the algorithm seeks for the Q-tensor field that renders the Landau-de Gennes energy functional stationary. The Landau-de Gennes formalism makes use of a phenomenological continuum theory model for the LC. The total free energy of the system in the Landau-de Gennes formalism can be written as a sum of the elastic distortion energy density, the thermotropic (or Landau) energy density, the external field induced energy density and the surface energy density appearing at interfaces.

Since no electric or magnetic fields are applied, the external-field-induced energy density is zero. Strong anchoring at the surfaces is assumed, so that also the surface energy density term disappears. The elastic and dielectric properties of E7 are used in the simulations (K11 = 11.1 $\mathrm{pN}, \mathrm{K} 22=6.5 \mathrm{pN}, \mathrm{K} 33=17.1 \mathrm{pN}$ ) together with some characteristic values for the bulk thermotropic coefficients. Very few measurements of thermotropic coefficients are found in the literature so we use thermotropic coefficients based on the ones measured for $5 \mathrm{CB}$. We use thermotropic coefficients based on the ones measured for $5 \mathrm{CB}$ at a reduced temperature of $-2^{\circ} \mathrm{C}$, giving rise to an equilibrium order parameter of 0.54 . To increase the natural length scale of variations in the order parameters and to obtain faster numerical convergence we use 10 times smaller values for the thermotropic coefficients. 